\title{
La biblioteca de Alí Bey
}

\author{
Patricia Almarcegui \\ Universidad de Zaragoza
}

Resumen

En 1795 el viajero Domingo Badía Leblich, Ali Bey, abandonaba la ciudad de Córdoba debido a problemas económicos. La construcción de un globo aerostático le había llevado a la ruina. Como último recurso, ponía a la venta parte de su biblioteca, la única prueba que existe de las fuentes que configuraron su Plan de viaje al África y el testimonio de la formación ilustrada del viajero. Entre otras cosas, esta lista de libros pone en evidencia cómo a las principales investigaciones que se han realizado sobre Badía, que lo sitúan como precursor de los intereses coloniales de España en África y la imagen perfecta del personaje fascinado por Oriente, hay que añadir el que fue objetivo principal del viajero ilustrado europeo: completar con la experiencia práctica del viaje el saber teórico adquirido y proyectar los conceptos civilizatorios en las geografías visitadas.

A finales de 1795, aquél que fuera viajero, arbitrista, espía, narrador, inventor, príncipe, grabador, bibliotecario, dramaturgo, orientalista, astrónomo, articulista, geógrafo, usurpador de identidades e ilustrado catalán, Domingo Badía y Leblich (1767-1818) abandonaba Córdoba. A su salida, dejaba en manos de un amigo la única prueba de la existencia de su biblioteca que se ha encontrado hasta la fecha y que nunca antes había sido publicada. Una lista con 48 referencias a obras o autores, titulada «Libros y música que existe en poder de D. Felipe Josef Benítez Reyes», en la que la parte relativa a la música aparece

Cuadernos de Estudios del Siglo XVIII, núms. 10-11. Oviedo, Instituto Feijoo de Estudios del Siglo XVIII, Universidad de Oviedo, 2002, págs. 5-16. 
en blanco. Esta lista se encuentra dentro del volumen llamado Manuscrits copias, vol. I, 1/III/1802, págs. 158-170 (copias manuscritas de documentos biográficos sobre Badía) y pertenece a la colección ${ }^{1}$ que el diplomático Eduard Toda y Güell fue adquiriendo sobre el viajero a lo largo de su vida, una colección que fue legada a su muerte al Archivo Histórico de la Ciudad de Barcelona. Como se verá más adelante, estas identificaciones confirman que Badía fue un heredero fiel de la tradición ilustrada de su época, situado a la altura de muchos de los hombres de ciencia dieciochescos europeos.

En la ciudad de Córdoba el futuro viajero desempeñó el cargo de Administrador de la Real Renta de Tabaco durante tres años, y consiguió aplicar y publicar los diferentes estudios que había realizado hasta entonces. Antes de su llegada, había acompañado a su padre en el traslado de Barcelona (1774) a Vera (Almería) para ejercer el puesto de Administrador de Utensilios de la Costa de Granada. Un tiempo después, cuando Badía tenía 18 años, heredaba este cargo y llegaba a ascender a Contador de guerra de la ciudad. En Vera el futuro viajero tuvo sus primeros contactos con el mundo árabe. En un lugar en el que bereberes, árabes... fueron percibidos, probablemente tratados y su idioma escuchado, se empezó a conformar su imaginario oriental, pues esta población constituía uno de los lugares de intercambio mercantil y cultural entre el sur de España y el norte marroquí en el que se aprovisionaban las tropas españolas destinadas en Ceuta y Melilla.

Badía se había trasladado a Córdoba con su familia. Con él iban su mujer, oriunda de Vera, Maria Lucía Burruezo Campoy (nacida el 17/VIII/1767), su hijo Pedro (6/VII/1792) y allí nació dos años más tarde su hija María Asunción (10/VIII/1794). Una vez situado económica y socialmente, comenzó a rentabilizar y dar a conocer públicamente sus estudios, de forma que escribió y editó algunos trabajos que mostraban sus conocimientos e intereses por las ciencias física, química y matemáticas, muchos de los cuales serían aplicados años más tarde en buena parte de las observaciones científicas de sus Viajes. Como por ejemplo: las observaciones meteorológicas realizadas mientras paseaba al sureste de la ciudad ${ }^{2}$, la memoria sobre la navegación aérea ${ }^{3}$, el problema sobre la coagulación de la mezcla del aceite y el agua ${ }^{4}$, las observaciones físicas sobre el vacío de la máquina neumática ${ }^{5}$, la propuesta para fabricar una olla que eco-

\footnotetext{
${ }^{1}$ Esta colección está formada por los volúmenes siguientes: Alí Bey, Manuscrits copias, vols. I-II, Alí Bey, Documents originals, vols. I, II y IV, Alí Bey, Memorias originals, vol. III.

2 Alí Bey, Manuscrits copias, vol. I, 14/X/1793, pág. 38.

${ }^{3}$ Ibidem, pág. 92.

${ }^{4}$ Ibidem, págs. 178-83.

5 Ibidem, págs. 184-93.
} 
nomizase carbón y leña ${ }^{6}$, la fórmula matemática necesaria para la ascensión del globo aerostático ${ }^{7}$ y una propuesta de mejora en la medidas de las observaciones meteorológicas ${ }^{8}$. Todas estas investigaciones culminaron en un proyecto en el que había invertido más de tres años y significó, posiblemente, la empresa más compleja que había realizado hasta el momento: la construcción de un globo aerostático. Por otro lado, este empeño demostró que parte de los propósitos y obsesiones que incluiría más tarde en las revisiones de su Plan de viaje al África con objetos políticos y científicos (8/IV/1801) ya se encontraban en sus proyectos anteriores, pues una de las finalidades del globo sería el uso militar. Sin embargo, su ejecución nunca llegó a llevarse a cabo. Desde el 19 de mayo de 1795, fecha en la que se comenzó a construir «El Guadalupe» y se instaló en el Campo de la Merced, hasta el 4 de julio, en que el Consejo de Castilla obligó a suspender toda operación hasta nueva orden, se hicieron cinco intentos vanos para que volase ${ }^{9}$. Paradójicamente, el Consejo aceleró la desautorización del ascenso, cuando recibió una nota del padre de Badía en la que mostraba su temor ante el peligro que suponía la inflamación del gas del globo para la vida de su hijo. El viajero hizo todo lo que estuvo en su mano para que se le remitieran los documentos originales en los que se había basado el Consejo para su prohibición y así poder enviarlos a las Reales Escuelas de Física y Química para que decidieran sobre la viabilidad o no del proyecto. Pero dichos informes nunca le fueron remitidos, y el globo y los instrumentos que había traído con tanto cuidado desde Inglaterra para sus observaciones atmosféricas —en lo que parece ser su primer viaje a este país- se mantuvieron inutilizados en el Campo de la Merced. Badía intentó conseguir varias veces la licencia, pero jamás llegó a ser aprobada. A toda esta serie de percances se añadió la deuda económica que había contraído con sus suegros y amigos, quienes habían subvencionado su proyecto, y las enemistades que se había granjeado con una parte de la población cordobesa, debido a causas no muy claras, pero vinculadas probablemente

\footnotetext{
${ }^{6}$ Ibidem, pág. 282.

${ }^{7}$ Diario de Madrid, 5/VI/1795.

${ }^{8}$ Diario de Madrid, 29 y 30/ VI/1795.

${ }^{9}$ Vid. sobre el tema de la construcción y la voladura del globo aerostático, Alí Bey, Manuscrits copias, vol. I, págs. 40-129; Alí Bey, Documents originals, vol. IV, pp. 11-25; «A los Suscriptores del Globo Aerostático», en ibidem, 6/X/1795, interpolado entre los folios 23 y 24 (existe un tomo de este impreso en la biblioteca de Antonio Cánovas del Castillo, actualmente en la biblioteca del Museo Lázaro Galdeano); Augusto Casas, Ali-Bey. Vida, viajes y aventuras de Don Domingo Badía, Barcelona, Luis Miracle, 1943, págs. 21-3; Ramón Ezquerra, «Domingo Badia: sus audaces viajes y proyectos», en Archivos del Instituto de Estudios Africanos I, 1947, pág. 110; Alí Bey, Viajes por Marruecos, ed. de Salvador Barberá, Barcelona, Ediciones B, 1997, pág. 15; Domingo Badía (Alí Bey), Ali Bey en Marruecos, ed. de Celsa C. García Valdés y Michael McGaha, Pamplona, EUNSA, 1999, pág. 13.
} 
a su labor como ilustrado y su quehacer como covachuelista ${ }^{10}$. En estas circunstancias, no tuvo más remedio que abandonar Córdoba.

No obstante, existen algunos documentos que demuestran que, unos meses antes, por la razón que fuera, ya había intentado dejar la ciudad y cambiar de ocupación. En febrero había solicitado una permuta de trabajo con Antonio López de Ochoa para ejercer el cargo de Teniente de la ronda montada del resguardo general de rentas de Sanlúcar de Barrameda ${ }^{11}$. Según Badía, este lugar poseía un clima más acorde con lo que parecían sus problemas de salud. Sin embargo debió de estar muy poco tiempo en este trabajo, ya que ese mismo año fue destinado a Puerto Real (Cádiz) ${ }^{12}$. Poco después, y continuando en su labor de ilustrado, ingresó en la Real Sociedad Sevillana de Amigos del País y la Real Sociedad Patriótica de Murcia, con la voluntad de participar más activa y socialmente en la mejora del bien público.

En noviembre de 1797 aparecía la primera noticia de su traslado a Madrid. Según el viajero, su llegada era debida a la voluntad de tramitar personalmente la indemnización que el Consejo de Castilla le debía tras haber suspendido la licencia del ascenso del globo. En su nuevo destino no se olvidó de las deudas que había contraído con sus familiares, e intentó por todos los medios que su cargo de Teniente del resguardo de Rentas Unidas de Puerto Real, que había abandonado, pasara a ser desempeñado por su suegro; de manera que, por un lado, pudiera aliviarle de la ruina que le había causado la subvención de la construcción del aerostático y, por otro, ayudarle en la manutención de su mujer e hijos, que se habían quedado a su cargo ${ }^{13}$. Sin embargo, esta petición fue revo-

${ }^{10} \mathrm{Vid}$. sobre sus relaciones cordobesas, Manuscrits copias, vol. I, agosto 1795, p. 112; Manuscrits copias, vol. II, 1796, págs. 1- 44 e ibidem, 20/I/1796, págs. 45-67; Ramón Ezquerra, op. cit., pág. 110; Salvador Barberá, op. cit, pág. 16; ed, de Celsa C. García Valdés y Michael McGaha, op. cit., págs. 13-4; Michael McGaha, «Domingo Badía (Ali Bey) en Marruecos», en Espacio, Tiempo y Forma IX, 1996, pág. 22.

${ }^{11}$ Manuscrits copias, vol. I, 6/II/1795, pág. 140. Ningún biógrafo de Badía ha recogido esta solicitud de permuta a Sanlúcar de Barrameda. Vid. por ejemplo: Augusto Casas, op. cit. pág. 20: «Después de algunos años fue promovido a la Comandancia principal del Resguardo del Puerto de Santa María; pero estando entonces ya dedicado a la preparación de sus famosos viajes, presentó la dimisión»; ed. de Celsa C. García Valdés y Michael McGaha, op. cit., p. 14: «Se le ofreció el puesto de teniente de resguardo de rentas unidas de Puerto Real, empleo muy inferior al que había desempeñado en Córdoba (...) Después de sólo dos meses en Puerto Real, pidió permiso para ausentarse del cargo durante un tiempo con el fin de ir a Madrid».

12 Ibidem, 1795, pág. 139. Vid. también la duda de Salvador Barberá respecto a este trabajo, op. cit., pág. 15 : «Según Casas, Badía partió al año siguiente de Córdoba, trasladado a Puerto Real (Cádiz). Tal vez no llegó a tomar posesión de ese destino, pues omite su mención en los curricula [Documents originals, vol. II, 1807, pág. 171] que redactó posteriormente al término de su viaje».

${ }^{13}$ Manuscrits copias, vol. I, 15/VI/1798, pág. 145: «Últimamente viendo arruinados a mis infelices padres políticos por esta causa (pues eran mis fiadores) he solicitado pase a ellos mi empleo para que puedan alimentarse aquellos desdichados ocho individuos que hoy día están encerrados en un cuarto comiendo escasamente de la limosna que hace un amigo mío cuya caridad no podrá extenderse a muchos días (...) La sola demora en este asunto debe acarrear las mas funestas consecuencias, estando aquellos desdichados en la situa- 
cada y parte de su estancia y movimientos en la capital los dedicó a intentar pagar a sus acreedores de Córdoba ${ }^{14}$. Un año después, el viajero se instalaba en el número 33 de la calle de la Puebla (18/X/1799) y en la casa $n^{\circ} 2$, manzana 552 de la calle de Leganitos ${ }^{15}$ (27/V/1800). Mientras tanto, pudo mantenerse económicamente gracias al trabajo de D. Pablo Sangro y de Merode, príncipe de Castelfranco ${ }^{16}$, a quien sirvió como secretario y bibliotecario, lo que le permitió estar al tanto y consultar buena parte de las novedades editoriales que se publicaban en Europa.

En esta etapa madrileña Badía debió de realizar amplias lecturas y aproximaciones científicas que iría añadiendo al proyecto de sus Viajes y lo configurarían; pero, desgraciadamente, apenas quedaron pruebas de éstas en sus documentos. Sólo alguna muestra de aquellas obras que no pudo comprar: «Durante el tiempo de su permanencia en Madrid Badía recorrió todas las Bibliotecas (...) Veía también los libreros de la calle de Carretas, llevando nota de los libros franceses que recibían y que por escasez de recursos no podía comprar. Casi todas las obras se refieren a ciencias físicas o matemáticas ${ }^{17}$ ». Y de aquéllas de las que tuvo que deshacerse; es decir, el listado de obras anteriormente referido, y la única muestra que ha quedado de los libros que formaron su biblioteca. Gracias a ella, se puede conocer alguna de las fuentes de la parte política y científica de sus viajes (esta última nunca llegaría a redactarse, aunque existen algunos apuntes diseminados en los Documentos originales).

Todos estos libros fueron encomendados a su amigo de Puerto Real, Felipe José Benítez Reyes, con la voluntad de que los fuera vendiendo y pudiera pagar a parte de los acreedores que había dejado en Córdoba.

La identificación de sus títulos se ha realizado consultando los catálogos siguientes: Aguilar Piñal, F., Bibliografía de autores españoles del siglo XVIII (FAP), Brunet, Jacques-Charles, Manuel du libraire et de l'amateur de livres (JCB) y el National Union Catalogue (NUC). La clasificación y numeración se ha hecho por orden alfabético, y se han citado las primeras ediciones de cada uno de los libros. En el apéndice se ha reproducido el orden original de las referencias a obras y autores de la copia manuscrita.

Los libros que Badía poseía en su biblioteca eran:

ción mas horrible, quebrantada su salud, especialmente los niños, siendo su único alimento un infeliz pan de maíz».

${ }^{14}$ Vid. sobre este tema, ibidem, sin fecha, págs. 160-1 y 2/III/1799, págs. 164-7.

${ }^{15}$ Ibidem, sin fecha, pág. 160.

${ }^{16}$ Documents originals I, p. 68 v. Vid. también sobre su trabajo como bibliotecario, ed. de Celsa C. García Valdés y Michael McGaha, op. cit., p. 14; Salvador Barberá, op. cit., p. 18; Augusto Casas, op. cit., p. 23. Carlos Soldevila, Figures de Catalunya, Barcelona, Aedos, 1955, p. 174.

${ }_{17}$ Manuscrits copias, 1799, p. 162. 
1. Almeida, Teodoro de, Cartas físico-matemáticas de Teodosio a Eugenio: complemento de la recreación filosófica que escribió el P. D. Teodoro de Almeida. Esta obra contiene un aparato de principios necesarios para entender la física experimental, como se explica en los Reales Estudios de S. Isidro. Traducida al castellano por el Doctor D. Francisco Girón y Serrado, Madrid, 1782, 2 vols. (FAP, vol. IV, ${ }^{\circ}$ 1479).

2. Almeida, Teodoro de, Recreación filosófica o diálogo sobre la filosofía natural para instrucción de personas que no han frecuentado las aulas. Traducido del portugués por D. Luis Antonio Figueroa, Madrid, 1792, 8 vols. (FAP, vol. III, n $\left.\mathrm{n}^{\circ} 3854\right)$.

3. Ballexserd, Jacques, Disertation sur l'éducation physique des enfans, depuis leur naissance jusqu'à l'âge de puberté, París, 1762 (NUC, vol. XXXII, p. 509).

4. Baumé, Antoine, Élémens de pharmacie théorique et pratique: contenant toutes les opérations fondamentales de cet art, avec leur définition, \& une explication de ces opérations, par les principes de la chymie, París, 1762 (NUC, vol. XL, p. 53).

5. Bergman, Olof Tobern, Dissertatio chemica de analysi aquarum frigdarum, Upsala, 1788 (NUC, vol. XLVIII, p. 424).

6. Bertier, Joseph-Étienne, Physique du ciel, où l'on confronte sans partialité le vuide avec l'éther, l'attraction avec l'impulsion. Et où sont contenus les principes de toute le physique généralement, París, 1763, 3 vols. (NUC, vol. L, p. 439).

7. Caille, Nicolas Louis de la, Cours élémentaire et complet de mathématiques pures, París, 1794-95 (NUC, vol. CCCX, p. 327).

8. Calepino, Ambrosio, Bergomatis Eremitani Dictionarium, Reggio, 1502 (NUC, vol. LXXXIX, p. 634).

9. Condillac, Étienne Bonnot de, La Logique ou les premieres développements de l'art de penser, París, 1780 (NUC, vol. CXVIII, p. 512).

10. Descripción de las máquinas de más generalmente utilidad que hay en el Real Gabinete de ellas, establecido en el Buen Retiro, hecha de orden de S. M., Madrid, 1798 (FAP, vol. IX, $\mathrm{n}^{\circ}$ 1652).

11. Feijoo y Montenegro, Fray Benito Jerónimo, Ilustración apologética al primero y segundo tomo del teatro crítico, donde se notan más de cuatrocientos descuidos al autor del antiteatro; $y$ de los setenta, que éste imputa al autor del teatro crítico, se rebajan los sesenta nueve y medio, Madrid, 1729 (FAP, vol. III, n ${ }^{\circ}$ 1892).

12. Fénelon, François de Salignac de la Mothe, Suite du quatrième livre de l'Odyssée d'Homère, ou les Avantures de Télémaque, fils d'Ulisse, París, 1699 (NUC, vol. CLXIX, p. 331). 
13. Foronda, Valentín de, Lecciones ligeras de química. En que se trata del calórico, de los fluidos elásticos, de las afinidades o atracciones, de la nueva nomenclatura química, de las sustancias simples, de los álcalis de los ácidos, Madrid, 1791 (FAP, vol. III, nº 4390).

14. Fourcroy, A.-F., Élémens d'histoire naturelle et de chimie, París, 1782 (FAP, vol. CVXXIX, p. 313).

15. Fugère, A. C. y Goguet, Antoine Yves, De l' origene des loix, des arts, et des sciences; et de leurs progrès chez les anciens peuples, París, 1758 (NUC, vol. CCIV, p. 238).

16. Gumilla, José, El Orinoco ilustrado, historia natural, civil y geográfica de este gran río, y de sus caudalosas vertientes: gobierno, usos, y costumbres de los indios sus habitadores, con nuevas, y útiles noticias de animales, árboles, frutos, aceites, reseñas, hierbas, y raíces medicinales: y sobre todo, se hallarán conversiones muy singulares a nuestra santa fe y cosas de mucha edificacion, Madrid, 1741, 5 vols. (NUC, vol. CCXXIII, p. 247).

17. Guyton de Morveau, Louis Bernard, Elémens de chymie, théorique et pratique, rédigés dans un nouvel ordre, d'après les découvertes modernes pour servir aux Cours publics de l'Académie de Dijon, Dijon, 1777, 3 vols. (NUC, vol. CCXXIV, p. 231).

18. Hervás y Panduro, Lorenzo, Idea dell'universo, Cesena, 1778-87, 21 vols. (NUC, vol. CCXLIII, p. 342).

19. Huarte de San Juan, Juan, Examen de ingenios para las ciencias en el cual el lector hallará la manera de ingenio para escoger la ciencia en que más ha de aprovechar: y la diferencia de habilidades que hay en los hombres, y el género de letras y artes que a cada uno responde en particular, Amberes, 1573 (JCB, vol. III, p. 357).

20. Ibáñez de la Rentería, José Agustín, Fábulas en verso castellano, Madrid, 1789 (FAP, vol. IV, nº 3530).

21. Journal des Savants, París, 1665-1792 (JCB, vol. VI, p.1853).

22. Luján y Suárez de Góngora, Pedro Francisco (Duque de Almodóvar), Década epistolar sobre el estado de los libros en Francia, Madrid, 1781 (FAP, vol. V, $\mathrm{n}^{\circ}$ 1853).

23. Mauduyt de la Varenne, Pierre Jean Claude, Mémoire sur les différentes manières d'administrer l'électricité et observations sur les effets qu'elles ont produits, París, 1784 (NUC, vol. CCCLXX, p. 263).

24. Maur, Carlos, Elementos de matemática pura, Madrid, 1778, 2 vols. (FAP, vol. $\left.\mathrm{V}, \mathrm{n}^{\circ} 664\right)$.

25. Montengón, Pedro, El Antenor, Madrid, 1788, 2 vols. (NUC, vol. CCCVIIIC, p. 67). 
26. Montengon, Pedro, El Rodrigo, romance épico, Madrid, 1793 (NUC, vol. CCCVIIIC, p. 68).

27. Montengon, Pedro, Eudoxia, hija de Belisario, Madrid, 1793 (NUC, vol. CCCVIIIC, p. 67).

28. Niebuhr, Carstens, Beschreibung von Arabien aus eigene Beobachtungen und im Lande selbst gesammleten Nachrichten abgefasset, Copenague,1772, 3 vols. (NUC, vol. CDXVIII, p. 612).

29. Philalethes, Eirenaeus (pseudónimo), Introitus apertus ad occlusum regis, Ámsterdam, 1667 (NUC, vol. CDXLV, p. 258).

30. Regnault, Noël, Les Entretiens physiques d'Ariste et d'Eudoxe, ou Physique nouvelle en dialogues qui renferme précisement ce qui s'est découvert de plus utile dans la natur, Amsterdam, 1732, 3 vols. (NUC, vol. CDLXXXV, p. 687).

31. Regnault, Noel, L'Origine ancienne de la physique nouvelle, París, 1734 (NUC, vol. CDLXXXV, p. 687).

32. Sadoleto, Jacopo, De laudibus philosophiae, Lyon, 1538, 2 vols. (NUC, vol. DXIII, p. 590).

33. Saint-Remy, Surirey de, Mémoires d'artillerie, París, 1745, 3 vols. (NUC, vol. DXV, p. 275).

34. Samaniego, Félix María, Fábulas en verso castellano para uso del Real Seminario Vascongado de los Amigos del País. Publicados por orden por la misma sociedad, Valencia, 1781-84, 2 vols. (FAP, vol. VII, $\mathrm{n}^{\circ} 3023$ ).

35. Saussure, Horace-Benedict de, Voyage dans les Alpes, précédés d'un essai sur l'histoire naturelle des environs de Genève, Neuchâtel, 1780-96, 4 vols. (NUC, vol. DXXII, p. 92).

36. Saverien, Alexandre, Histoire des mathematiciens, París, 1761 (NUC, vol. DXXII, p. 301).

37. Sigaud-Lafond, Joseph Aignan, Élemens de physique théorique et expérimentale pour servir de suite á la Description de l'usage d'un cabinet de physique expérimentale, París, 1787 (NUC, vol. DXLV, p. 650).

38. Solís, Antonio de, Historia de la conquista de México, Madrid, 1684 (NUC, vol. DLV, p. 458).

39. Sturm, Cristoph Christian, Betrachtungen über die Werke Gottes im Reiche der Natur und der Versehung auf alle Tage des Jahres, Frankfurt und Leipzig, 1780, 2 vols. (NUC, vol. DLXXV, p. 23).

40. Torrubia, José, Centinela contra Franc-Masones. Discurso sobre su origen, instituto, secreto y juramento. Descúbrese la cifra con que escriben, y las acciones, señales y palabras con que se conocen. Impúnanse con la Pastoral del Ilustrísimo Señor D. Pedro María Justiniani, Obispo de Vintimilla, Madrid, 1752 (FAP, vol. VIII, $\mathrm{n}^{\circ}$ 1074). 
41. Ulloa, A. P., Noticias americanas: entretenimientos físico-históricos, sobre la América meridional y la Septentrional oriental. Comparaciones generales de los territorios, climas y producciones de las tres especies, vegetales, animales y minerales: con relación particular de las petrificaciones de cuerpos marinos de los indios naturales de aquellos países, sus costumbres y usos. De las antigïiedades: discurso sobre la lengua y sobre el modo en que pasaron los primeros pobladores, Madrid, 1772 (FAP, vol. VIII, ${ }^{\circ}$ 1621).

42. Vitoria, Baltasar de, Teatro de los dioses de la gentilidad, Madrid, 1676, 2 vols. (NUC, vol. DCXL, p. 207).

43. Wolff, Christian, Compendius elementorum matheseos universae, in usum studiosae iuventutis adornatum, Laussane, 1757, 2 vols. (NUC, vol. DCLXXI, p. 186).

A parte de todos estos títulos, el viajero tenía tres obras que no se han podido identificar:

44. Una lógica

45. Un libro sobre el uso de las esferas y los globos aerostáticos

46. Un oficio de la Semana Santa de Pascua en latín y castellano

$\mathrm{Y}$ tres obras cuyas referencias bibliográficas no se han podido completar:

47. La compilación de narraciones orientales, Mil y un días

48. Y el Compendio de la Historia de España, escrita en francés por R. P. Duchesne, Amberes, 1758, 2 vols., y traducida por José Francisco Isla (FAP, vol. VIV, $n^{\circ}$ 4336). Falta la primera edición.

En total, entre estos libros había: un $22,9 \%$ dedicados a la literatura (números: $11,12,19,20,22,25,26,27,34,42,47) .10,4 \%$ a las matemáticas $(1,7$, $24,36,43)$.

$10,4 \%$ a la filosofía $(2,9,32,44) .8,3 \%$ a la química $(5,13,14,17) .8,3 \%$ a los viajes $(16,28,35,41) .8,3 \%$ a la física $(6,30,31,37) .4,1 \%$ a la historia $(38,48) .2 \%$ a la astronomía (18). $2 \%$ a la construcción de máquinas (10). $2 \%$ a la educación física (3).

$2 \%$ a la lengua latina (8). $2 \%$ a la farmacia (4). $2 \%$ a la alquimia (29). $2 \%$ a las leyes jurídicas (15). $2 \%$ a la electricidad (23). $2 \%$ a las armas (33). $2 \%$ a la masonería (40). $2 \%$ a los oficios religiosos católicos (46). $2 \%$ a la observación de la naturaleza (39). $2 \%$ a la prensa (21) y $2 \%$ a la construcción de globos y esferas (45). Según esta clasificación, la lecturas más realizadas por Badía fueron las literarias, lo que confirmaría las posteriores aproximaciones que a esta materia realizó en su escritura, como fueron: varias traducciones, la composición de una tragedia y su proyecto para publicar un Diario de los teatros. Las lectu- 
ras de obras matemáticas, filosóficas, químicas y físicas muestran su formación autodidacta (sólo estuvo inscrito, alrededor de 1796, en la Real Academia de Bellas Artes de S. Fernando y las Reales Escuelas de Química y Física). Por otro lado, el hecho de poseer en su biblioteca el libro del viajero danés Niebuhr, que citará en su posterior Plan de viaje al África de 1801, así como otros libros de viajes, demuestra que comenzó a trazar su proyecto durante su estancia en Vera y no en su posterior viaje a Madrid, tal y como siempre se ha señalado. Y en fin, la dispersión temática de sus restantes lecturas señalan que Badía poseyó una nómina representativa de obras que lo acercan, incluso incluyen, en parte de algunos de los ilustrados españoles más significativos. Y no sólo por estas obras, sino por el número de trabajos de tipo arbitrista, científico, orientalista... que realizó a lo largo de su vida, los cuales demostraron su amor a la verdad y a las virtudes de la ciencia. El viajero intentó enjuiciar críticamente aquella geografía visitada y reducirla al discurso de la razón. Y demostró cómo el viaje se había transformado, en su imagen de hombre del XVIII, en una suerte de investigación comparada, donde el juicio debía ser suspendido hasta que la evidencia demostrara su veracidad y lo que percibía debía reducirse a los conocimientos adquiridos antes de su partida. Sin embargo, esto no fue del todo posible. Y subyugado en el transcurso de su viaje por el nuevo paisaje y, mientras revisaba y preparaba su edición francesa, ocho años después de su vuelta, se vio seducido y emocionado por aquel espacio, hasta llegar a compartir algunas de las premisas del viajero romántico: «La sensación que experimenta el hombre que por primera vez hace esta corta travesía, no puede compararse sino al efecto de un sueño. Al pasar en tan breve espacio de tiempo a un mundo absolutamente nuevo y sin la más remota semejanza con el que acaba de dejar, se halla realmente como transportado a otro planeta ${ }^{18}$ ». De tal manera que, finalmente, en la escritura de Domingo Badía y Leblich se fueron articulando las premisas del viajero ilustrado y las del romántico europeo.

${ }^{18}$ Salvador Barberá, op. cit., p. 133. 
APÉNDICE. Lista de libros y música que existe en poder de don Felipe Josef Benítez en Cádiz ${ }^{19}$

Madrid $1^{\circ}$ de Mayo de 1802, Domingo Badía y Leblich. Queda en poder de D. Felipe Benítez en Puerto Real.

Libros en un cajón:

$\begin{array}{clr}\text { Volúmenes } & \text { Reales } \\ 8 & \text { Almeida, Recreaciones filosóficas } & 104 \\ 2 & \text { Id, Cartas Físico Matemáticas } & 26 \\ 2 & \text { Nieburh, Viage a Arabia } & 20 \\ 3 & \text { Berties, Física del Cielo } & 39 \\ 4 & \text { Sturm, Reflexiones sobre la naturaleza } & 56 \\ 4 & \text { Espíritu de los Sabios de Trevoux } & 52 \\ 5 & \text { Regnault, Entretenimientos físicos } & 65 \\ 1 & \text { Manduit, Electrizidad Médica } & 14 \\ 2 & \text { Le Maur, Matemática pura } & 40 \\ 1 & \text { Saberien, Ciencias exactas } & 13 \\ 1 & \text { Bergman, Análisis de las aguas } & 15 \\ 1 & \text { Gumilla, Orinoco ilustrado } & 20 \\ 1 & \text { Foronda, Chímica } & 12 \\ 2 & \text { Wolffio, Compendio Matemático } & 14 \\ 1 & \text { Ulloa, Noticias Americanas } & 12 \\ 4 & \text { Hervás, Viage extático } & 52 \\ 1 & \text { Feijoo, Apología } & 8 \\ 1 & \text { Posibilidad de la Alchimia } & 3 \\ 3 & \text { Teatro de los Dioses } & 12 \\ 2 & \text { Solís, Conquista de México } & 8 \\ 1 & \text { Torrubia, contra Francmasones } & 2 \\ 1 & \text { Balexerd, Crianza física de los Niños } & 4 \\ 1 & \text { Semana Santa, Latín y Castellano } & 22 \\ 2 & \text { Antenor } & 28 \\ 1 & \text { Eudoxia } & 14 \\ 1 & \text { Rodrigo } & 14 \\ 1 & \text { Condillac, Lógica } & 13 \\ 3 & \text { Baumé, Chímica } & 30 \\ 5 & \text { Origen de las Leyes, Artes, y Ciencias } & 70\end{array}$

${ }^{19}$ Manuscrits copias I, 1/III/1802, p. 168-70. La lista de música aparece en blanco. 
Volúmenes

Reales

S. Remí, Artillería

30

2

Saussure, Viage a los Alpes

100

1

Chímica de Dijon

14

Lugdunense, Philosofía $\quad 50$

Década Epistolar

12

Sigaud de la Fond, Física

112

Samaniego, Fábulas

10

La Renteria, id.

10

Usos de las Esferas y Globos

3

Mil y un días

25

Discernimiento de Ingenios

8

Isla, Historia de España

12

La lógica

8

Real Gavinete de Máquinas 4

Telémaco 16

Fourcroy, Historia natural y Chímica $\quad 60$

La Caille, Matemáticas $\quad 60$

\begin{tabular}{llr}
1 & Calepino de Salas & 20 \\
3 & Regnault, origen de la Fisica & 36 \\
\hline
\end{tabular}

108

$\frac{36}{1372}$

Música (en blanco) 